\title{
Modeling the joint behaviour of electricity prices in interconnected markets
}

\author{
Troels Sønderby Christensen ${ }^{* \dagger \ddagger}$, Fred Espen Benth ${ }^{\S}$
}

\begin{abstract}
The liberalization of energy markets worldwide during recent decades has introduced severe implications on the price formation in these markets. Especially within the European day-ahead electricity markets, increased physical connections between different market areas and a joint effort on optimizing the aggregate social welfare have led to highly connected markets. Consequently, observing the exact same hourly day-ahead prices for two or more interconnected electricity markets in Europe happens frequently. This affects the modeling of such prices and in turn the valuation of derivatives written on prices from such market areas. In this paper, we propose a joint model for day-ahead electricity prices in interconnected markets composed of a combination of transformed Ornstein-Uhlenbeck processes. We discuss the properties of the model and propose an estimation procedure based on filtering techniques. Furthermore, the properties of the model reveal that analytical prices are attainable for e.g. forwards and spread options.
\end{abstract}

Keywords: Day-ahead electricity prices; Interconnected markets; Stochastic modeling; Derivative pricing.

JEL Classification: C3, G1, Q4.

\section{Introduction}

With the introduction of liberalized energy markets worldwide, increased interconnectedness between different market areas has emerged. The so-called market coupling mechanism has increased the degree of connectivity even more in the European electricity markets. The market coupling works by collecting bids and offers for trading electricity the following day from each market area. Then, based on a social welfare optimization, a single algorithm calculates optimal flows in the system given interconnector limitations for each hour of the following day and the corresponding electricity prices; hence, the name day-ahead prices (Epex Spot (2019)). Fig. 1 illustrates this procedure in a two-market setup. The prices labelled "Domestic price" are the prices set if the areas were not connected and no cross-border flows of electricity were present. The difference between the domestic prices are rather large in this case. Though, by the market coupling mechanism, the price spread between these two areas are minimized. In the case of

\footnotetext{
${ }^{*}$ Corresponding author. Email: troelsc@math.aau.dk

${ }^{\dagger}$ Department of Mathematical Sciences, Aalborg University, Skjernvej 4A, 9220 Aalborg Øst, Denmark

${ }^{\ddagger}$ Quantitative Analytics, Centrica Energy Trading, Skelagervej 1, 9000 Aalborg, Denmark

$\S$ Department of Mathematics, University of Oslo, PO Box 1053 Blindern, N-0316 Oslo, Norway
}

Date: 31 January 2020. 


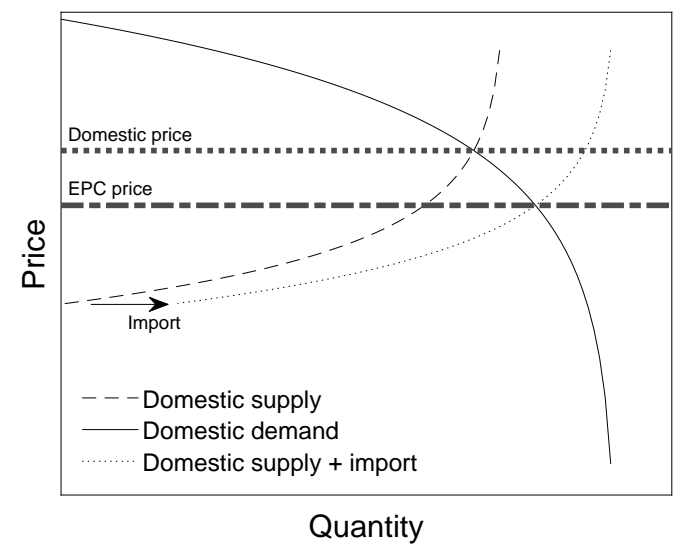

(a) High price area

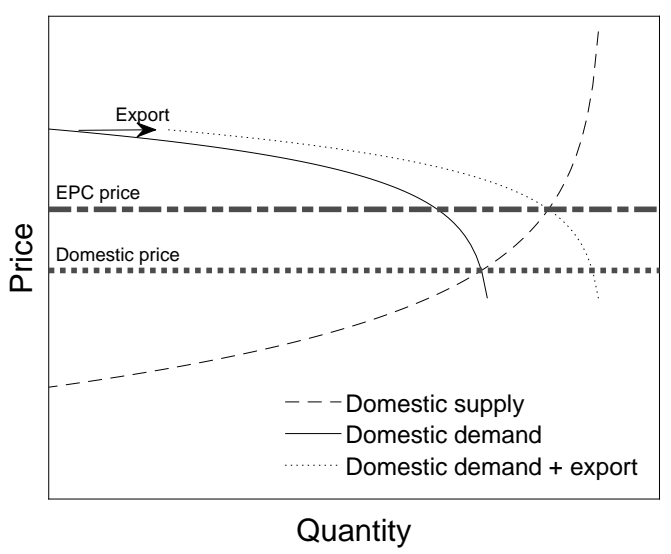

(b) Low price area

Figure 1: Example of market coupling in a two-market setup where exact price convergence is reached; i.e., the price spread between the two market areas is forced to be exactly zero by the market coupling mechanism. As a result, the domestic price in the high price area is reduced and the domestic price in the low price area is increased.

Fig. 1, we have enough interconnector capacity between the areas to eliminate the price spread completely. We define such a zero price spread as exact price convergence (EPC). The result is that cheap electricity is transferred from the low price area to the high price area, or loosely speaking, electricity flows from the area with a surplus of electricity to the area with a need for electricity.

As exemplified by Fig. 1, the market coupling has severe implications on the resulting prices in interconnected markets. Most notable is the presence of EPC that naturally has an impact on derivative prices, and in particular on transmission rights. A transmission right is basically a spread option between two connected market areas with the underlying being the spread between the market areas under consideration ${ }^{\dagger}$. When EPC occurs, the underlying spread is zero, and the transmission right becomes worthless. To conduct sensible pricing of transmission rights (and related derivatives in interconnected markets), a model capable of incorporating zero price spreads is therefore motivated.

Day-ahead electricity price modeling in general has been examined in numerous studies. To name a few, Lucia \& Schwartz (2002), Geman \& Roncoroni (2006), and Meyer-Brandis \& Tankov (2008) all focus on modeling the temporal dynamics of a single market. Lucia \& Schwartz (2002) exemplify their proposed model using daily average prices from the Nord Pool market, Geman \& Roncoroni (2006) use daily prices from the US, while Meyer-Brandis \& Tankov (2008) use EEX prices. Benth \& Šaltytè Benth (2004) and Benth et al. (2007) also focus on the temporal dynamics of a single market, and additionally discuss derivative pricing. Both of them present the model in the context of Nord Pool data, while the former also uses Brent oil data. Regarding the spatial interdependencies between different market areas, we mention Douglas \& Popova (2011) and Abate \& Haldrup (2017). Douglas \& Popova (2011) estimate a spatial error model on data from Pennsylvania-New Jersey-Maryland Interconnection, consisting of twelve market areas at the time of their investigation. Abate \& Haldrup (2017) investigate daily averages of

\footnotetext{
${ }^{\dagger}$ A transmission right can either be physically or financially settled, and will collapse if the physical transmission right is sold under the "use it or sell it" condition. We refer the interested reader to Mahringer et al. (2015) for more information.
} 
day-ahead prices from the markets making up the Nord Pool area, and find that the inclusion of spatial effects is as important as including temporal effects.

The literature concerning the joint modeling of day-ahead electricity prices for two or more interconnected markets where market coupling is taken into account is quite scarce. Mahringer et al. (2015) use a structural approach, where they model the supply and demand in a two-market setup that incorporates market coupling and allows for closed-form solutions for transmission rights. Pircalabu \& Benth (2017) propose a reduced-form model in a two-market setup defined in discrete time that includes the market coupling mechanism using a regime-switching model, but no closed-form solutions for derivative prices are given.

As Pircalabu \& Benth (2017) we propose a reduced-form model for day-ahead prices in a two-market setup that takes market coupling into account. Compared to Pircalabu \& Benth (2017), we differentiate us by formulating the model in continuous time. Instead of an ARMAGARCH copula formulation combined with a discrete Markov chain, we assume that day-ahead prices can be described by a suitable combination and transformation of Gaussian OrnsteinUhlenbeck processes. On one hand, this has the disadvantage that complicated dependence structures that are easily handled by copulas can be rather hard to incorporate in the present continuous-time formulation. On the other hand, the continuous-time formulation makes us able to derive closed-form solutions of forward and transmission right prices. Our contribution to the literature additionally includes an estimation procedure for the proposed model. It is based on two independent parts, namely a Kalman filtering part and a particle filtering part.

Although we in this paper have a European perspective, we believe that the results are interesting in other parts of the world as well where market area dependencies are present. As an example, Park et al. (2006) examine connected electricity markets in the US, and find that day-ahead prices in such connected market areas inherit spatial interdependencies. Also interesting is the work in Ignatieva \& Trck (2016), studying the dependence in day-ahead prices between different market areas in the Australian electricity market. Ignatieva \& Trck (2016) find a positive dependence between all market areas, but the strongest dependence is observed between adjacent market areas.

The remaining part of this paper is organized as follows: Sec. 2 introduces data and also serves as a motivation for the model choices made in later sections. In Sec. 3 we introduce the proposed model and discuss its properties. Sec. 4 concerns estimation of the model, while in Sec. 5 we exemplify the model empirically through a case study of German and French electricity prices and discuss derivative prices implied by the model. Sec. 6 concludes.

\section{Data presentation and model motivation}

This section presents data from the hourly power markets under consideration, and motivates the model choices made in later sections. The observations in this paper cover the three-year period from 1 July 2015 to 30 June 2018 and the market areas making up the Central Western Europe (CWE) area; Germany, France, the Netherlands, and Belgium. The market coupling algorithm was actually already introduced in the CWE area in November 2010; though, the underlying optimization algorithm was changed from the so-called available transfer capacity method to the flow-based market coupling method on 20 May 2015 (see Elia (2019)). In order to avoid results affected by the old methodology, we have thus chosen to fix our start date to 1 July 2015.

To give a sense of the frequency of how often EPC happens, Table 1 reports the observed percentage of time EPC occurred for each hour during the mentioned three years. The EPC percentage varies quite a bit depending on the chosen price spread and chosen hour. Fig. 2 illustrates the data showed in Table 1, and shows a clear pattern throughout the day for all 


\begin{tabular}{c|cccc}
\hline Hour & DE-FR & BE-FR & BE-NL & DE-NL \\
\hline 0 & 28 & 35 & 39 & 37 \\
1 & 30 & 40 & 42 & 37 \\
2 & 35 & 43 & 46 & 40 \\
3 & 41 & 47 & 49 & 44 \\
4 & 44 & 51 & 51 & 47 \\
5 & 43 & 51 & 49 & 47 \\
6 & 44 & 52 & 49 & 50 \\
7 & 42 & 50 & 47 & 49 \\
8 & 40 & 47 & 45 & 45 \\
9 & 28 & 37 & 36 & 33 \\
10 & 26 & 34 & 33 & 30 \\
11 & 23 & 32 & 31 & 28 \\
12 & 19 & 28 & 28 & 27 \\
13 & 21 & 30 & 29 & 26 \\
14 & 24 & 32 & 31 & 27 \\
15 & 31 & 39 & 36 & 33 \\
16 & 41 & 47 & 42 & 40 \\
17 & 44 & 49 & 47 & 44 \\
18 & 45 & 51 & 49 & 51 \\
19 & 41 & 48 & 45 & 48 \\
20 & 38 & 45 & 41 & 45 \\
21 & 36 & 43 & 42 & 4 \\
22 & 26 & 35 & 35 & 34 \\
23 & 15 & 26 & 27 & 27 \\
\hline & & & &
\end{tabular}

Table 1: Observed EPC percentage for each hour for the four different cross-border connections in Central-West Europe from 1 July 2015 to 30 June 2018.

spreads, with peaks around hour 5 and 18. The lowest values are reached at hour 12 and 23. Though, the rather high percentages in general suggests that EPC is important to consider in a modeling context in all cases.

Fig. 3(a) illustrates the evolution of the day-ahead electricity price in Germany and France for the hour from 21:00-22:00. In the rest of the paper, we denote the hour spanning the period 21:00-22:00 by hour 21 and likewise for the other hours. First of all, negative prices are observed, indicating oversupply of electricity in the grid. Traditional financial models that only allow nonnegative prices are therefore not able to take this feature into account. Even though it is only German prices that are negative for this particular time series, negative values are allowed for the other CWE market areas as well. Secondly, it is apparent that the mean and volatility of each price series seems to be highly seasonal. In particular, spring and summer periods result in a more calm behaviour, and autumn and winter periods in a more volatile behaviour, see Figs. 3(b)-3(c) that exemplify the seasonal differences.

Proceeding with the seasonal behaviour, Fig. 4 illustrates the day-ahead price differences between three chosen price pairs. We see that EPC happens more often during summer and spring than during autumn and winter in the FR-DE case in Fig. 4(a). A possible explanation for this is the fact that the cheap nuclear power production from France during summer levels out the differences between the French and German prices. Opposite, during winter the demand for electricity rises in France and in turn the French power prices rise, while the German prices 


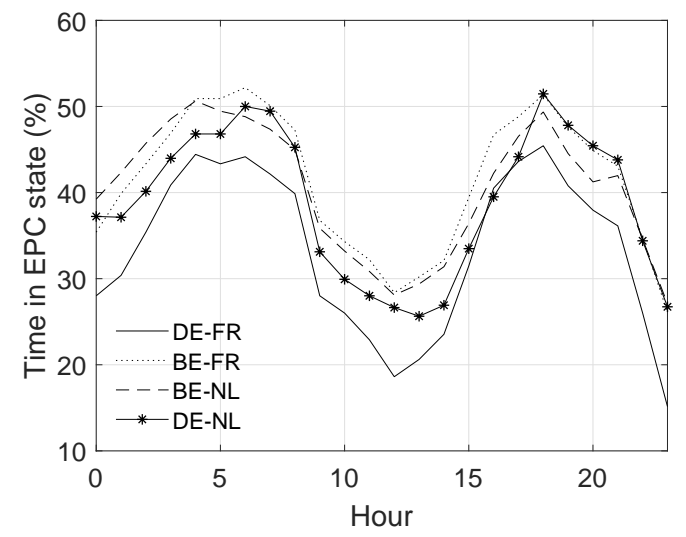

Figure 2: Time spent in the EPC state in percentage for each hour from 1 July 2015 to 30 June 2018 for the following spreads: DE-FR, BE-FR, BE-NL, and DE-NL.

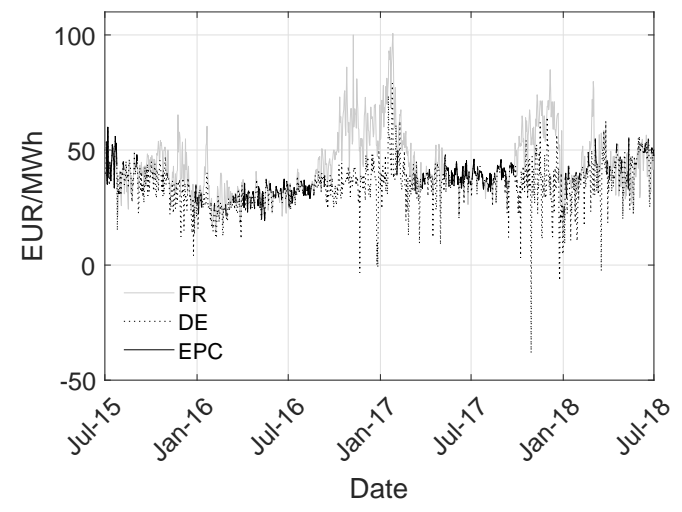

(a) Day-ahead prices for the entire period

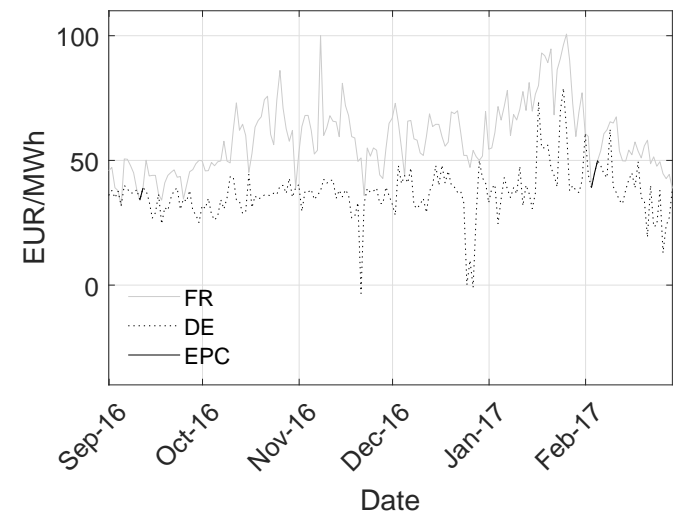

(b) Sub sample of autumn/winter day-ahead prices

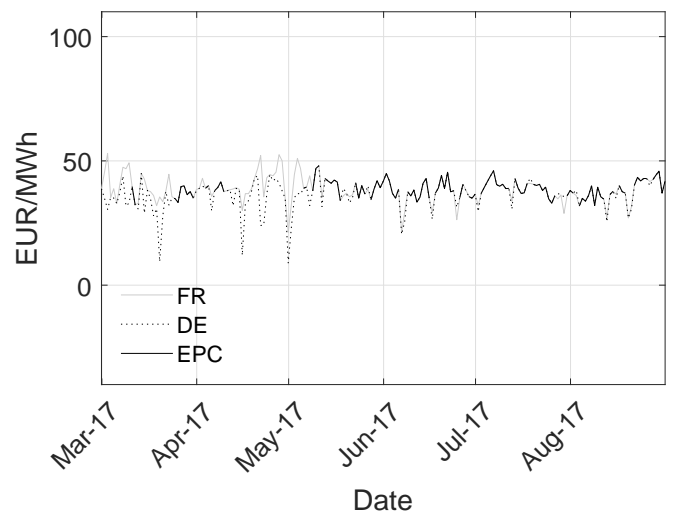

(c) Sub sample of spring/summer day-ahead prices

Figure 3: (a) Day-ahead pricse for France and Germany for hour 21 from 1 July 2015 to 30 June 2018, (b) and (c) are subsamples of the data presented in Fig. 3(a) to illustrate the differences between these periods. 


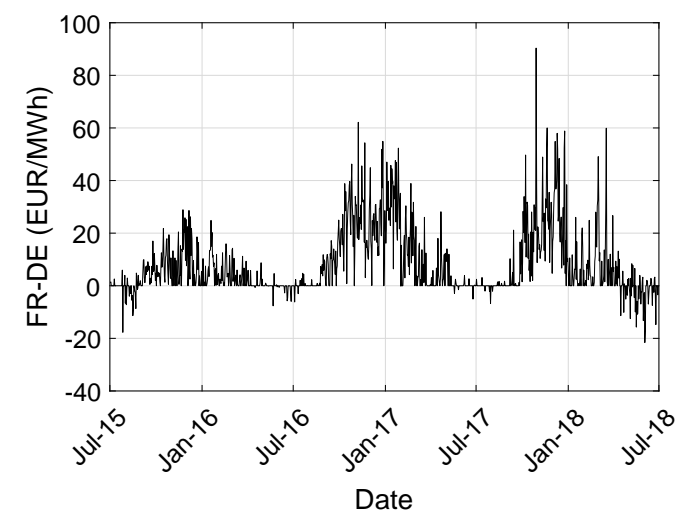

(a) FR-DE hour 21 day-ahead price spread

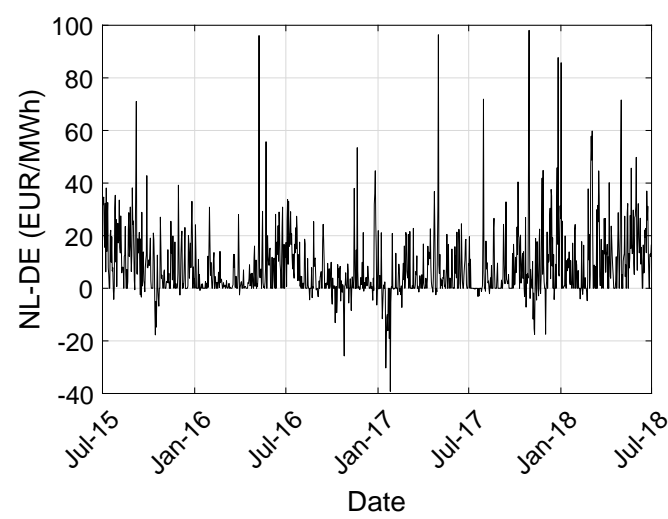

(b) NL-DE hour 12 day-ahead price spread

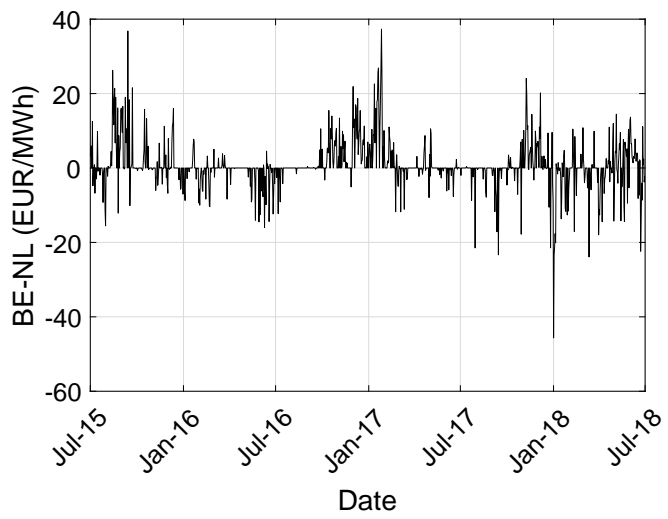

(c) BE-NL hour 8 day-ahead price spread

Figure 4: Day-ahead price differences for different hours and market areas.

do not rise similarly partly due to the high wind power production and the lower dependency on using electricity for heating, all in all making the gap between German and French prices widen during autumn/winter. With a physical connection between Germany and France being only of a certain size, and since the amount of needed transferred electricity between Germany and France to level out prices during autumn/winter is greater due to the larger price gap, congestion is observed more often during autumn/winter than spring/summer. A similar behaviour is observed in Fig. 4(c), while the rate at which EPC occurs seems more evenly spread out over the year in Fig. 4(b).

To investigate EPC in more detail, we plot in Fig. 5 the number of days being in the EPC state for the spreads considered in Fig. 4. The plot is constructed by dividing the observations in overlapping sub-samples of 60 days, and then calculating the number of EPC days in each sub sample. Fig. 5(a) shows a rather large difference between the time spent in EPC during spring/summer and autumn/winter, with a peak above $80 \%$ and the lowest value being $0 \%$. The same seasonal pattern is observed in Fig. 5(c), though with a lower amplitude. Opposite, the level is much more constant in the case of Fig. 5(b).

As is apparent from the above discussion, special features distinguish hourly day-ahead prices from traditional financial markets. Besides the obvious allowed negativity in the CWE area, seasonality in the mean level and volatility are known features of electricity prices (see e.g. 


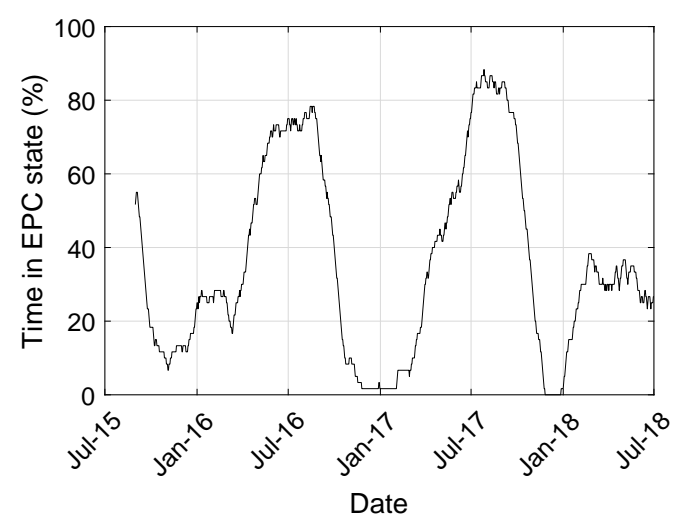

(a) FR-DE hour 21 day-ahead price

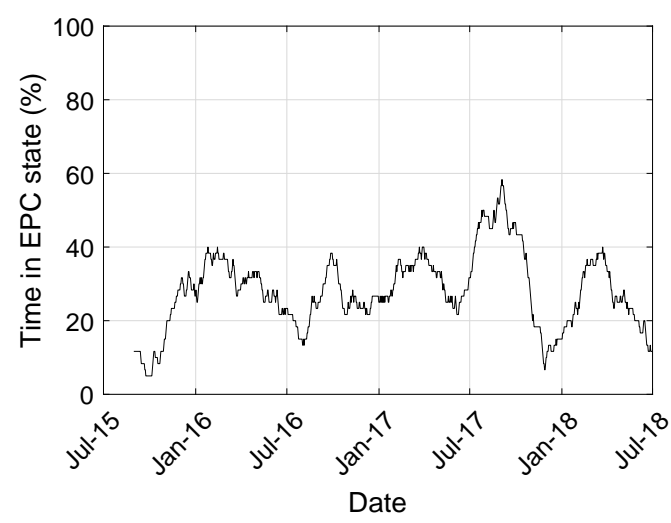

(b) NL-DE hour 12 day-ahead price

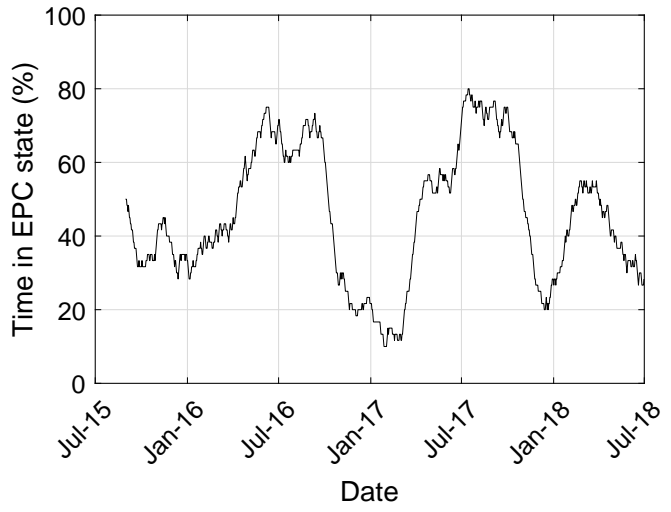

(c) BE-NL hour 8 day-ahead price

Figure 5: Time spent in the EPC state in percentage for overlapping sub-samples of 60 days. 
Haldrup et al. (2010), Pircalabu \& Benth (2017), Borovkova \& Schmeck (2017), or more recently the work of Benth et al. (2019) or Latini et al. (2019)), but as discussed above time-varying seasonality in probability of being in the EPC state is also important to take into account.

\section{Model description}

Let $(\Omega, \mathcal{F}, \mathbb{P})$ be a complete probability space. We propose the following model for the day-ahead prices

$$
S(t)=I(t) p(t)
$$

where $S(t) \in \mathbb{R}^{N}$ is the vector of day-ahead prices at time $t$ for $N$ market areas. The motivation for our model is to capture the price formation mechanism in a reduced-form manner. The exact convergence of day-ahead prices is represented by $I(t) \in \mathbb{R}^{N \times N}$, which is piecewise constant resembling the exact price convergence of prices from different market areas. An illustrative example of the process $I(t)$ is in the case $N=2$, where one can choose the states of $I(t)$ as

$$
I_{(1)}=\left[\begin{array}{ll}
w & 1-w \\
w & 1-w
\end{array}\right] \text { and } I_{(2)}=\left[\begin{array}{ll}
1 & 0 \\
0 & 1
\end{array}\right] \text {. }
$$

Here $w$ is a constant between zero and one that measures the pulling effect of each component of $S(t)$. In this example, state two - emphasized with subscript (2) - corresponds to no exact price convergence and $S(t)=p(t)$, whereas state one corresponds to exact price convergence with the common price being $w p_{1}(t)+(1-w) p_{2}(t)$. The states of $I(t)$ is driven by an underlying process $z(t)$, which we will discuss shortly.

The process $p(t) \in \mathbb{R}^{N}$ represents the partly latent non-convergence price set in each individual market area, and can thus be interpreted as the "domestic" prices. It is only partly latent, since we do in fact observe it in non EPC states. In the above example with $N=2$, we observe $p(t)$ whenever we are in state two. Based on the rather deterministic demand for electricity (which in turn is due to highly seasonal temperature patterns influencing the demand to a large extent) we assume that deviations from a deterministic seasonal function are temporary. We therefore assume that $p(t)$ can be divided in a deterministic part, $\Lambda(t) \in \mathbb{R}^{N}$ and a stochastic part $q(t) \in \mathbb{R}^{N}$, by

$$
p(t)=\Lambda(t)+q(t) .
$$

We assume that the stochastic part is governed by a multivariate Ornstein-Uhlenbeck process

$$
d q(t)=-K q(t) d t+\Sigma d B_{p}(t)
$$

where $K \in \mathbb{R}^{N \times N}$ is a constant matrix with the real part of its eigenvalues being positive, $\Sigma \in \mathbb{R}^{N \times N}$, and $B_{p}(t)$ is an $N$-dimensional Brownian motion. By this specification we allow for negative prices. While this is clearly unsatisfactory in traditional financial markets, negative prices in electricity markets do occur as discussed in Sec. 2.

We remark that a similar modelling idea has been proposed in a recent paper by Hinderks et al. (2018) in a structural Heath-Jarrow-Morton approach to electricity futures pricing. There, fixeddelivery futures price dynamics are modelled as a product of a noise process and a conditional expectation of a structural component. As for our model, the authors demonstrate the analytical tractability of such an approach in derivatives pricing.

The above specification holds for all $N$; however, we will limit ourselves to the case where $N=2$ in the rest of the paper for two reasons. First of all, an important feature of the model is 
the ability to price transmission rights. By construction, these derivatives are essentially spread options with the underlying being the spread between only two adjacent market areas. We thus argue that the case with $N>2$ is unnecessary regarding this application, but acknowledge that it could be relevant in other contexts. Secondly, modeling issues related to the underlying process governing $I(t)$ arises when $N>2$. We briefly comment on this in the next sections, where we elaborate on the components of the model in more detail.

\subsection{Properties of $p(t)$}

Let $\operatorname{vec}(\cdot)$ denote the vectorization operator that stacks the columns of a matrix $A$ on top of each other; i.e., if $A$ is an $m \times n$ matrix, then the vectorization operator applied on $A$ results in a column vector of size $m n \times 1$. Let further $\operatorname{vec}^{-1}(\cdot)$ be the operator satisfying $\operatorname{vec}^{-1}(\operatorname{vec}(A))=A$ for a square matrix $A$. Notice that for non-square matrices, $\operatorname{vec}^{-1}(\cdot)$ is not uniquely defined; however, since we only deal with square matrices, we omit a further discussion of this. Define the Kronecker sum between two square matrices $A$ and $B$ by $A \oplus B=A \otimes \mathbf{I}_{B}+\mathbf{I}_{A} \otimes B$, where $\otimes$ is the Kronecker product ${ }^{\dagger}$ and $\mathbf{I}_{B}$ is the identity matrix with the same size as $B$, and $\mathbf{I}_{A}$ is defined in the same way. We denote the filtration generated by the Brownian motion $B_{p}(t)$ by $\left\{\mathcal{F}_{t}^{B_{p}}\right\}_{t \geq 0}$. Prop. 1 states the distribution of $p(t)$ conditional on $\mathcal{F}_{s}^{B_{p}}$.

Proposition 1. Let $p(t)$ be defined as in Eq. (3), and let $s<t$. The distribution of $p(t)$ is normal conditional on $\mathcal{F}_{s}^{B_{p}}$ with mean

$$
\mathbb{E}\left[p(t) \mid \mathcal{F}_{s}^{B_{p}}\right]=\Lambda(t)+e^{-(t-s) K} q(s)
$$

and variance

$$
\operatorname{Var}\left[p(t) \mid \mathcal{F}_{s}^{B_{p}}\right]=\operatorname{vec}^{-1}\left((K \oplus K)^{-1}\left(\mathbf{I}-e^{-(t-s) K \oplus K}\right) \operatorname{vec}\left(\Sigma \Sigma^{\mathrm{T}}\right)\right)
$$

where $\mathbf{I}$ is the identity matrix of same size as $K \oplus K$.

Proof. The solution of Eq. (3) is seen to be

$$
q(t)=e^{-(t-s) K} q(s)+\int_{s}^{t} e^{-(t-u) K} \Sigma d B_{p}(u)
$$

by the multidimensional Ito formula. The normality and expected value follows. Using the Ito isometry, we get

$$
\operatorname{Var}\left[p(t) \mid \mathcal{F}_{s}^{B_{p}}\right]=\int_{s}^{t} e^{-(t-s) K} \Sigma \Sigma^{\mathrm{T}} e^{-(t-s) K^{\mathrm{T}}} d u
$$

For matrices $A, B$, and $C$ of appropriate sizes, two useful identities are $\operatorname{vec}(A B C)=\left(C^{\mathrm{T}} \otimes\right.$ $A) \operatorname{vec}(B)$ (see e.g. Macedo \& Oliveira (2013) and the references herein for more information on

\footnotetext{
${ }^{\dagger}$ The Kronecker product between two matrices $A \in \mathbb{R}^{m \times n}$ and $B \in \mathbb{R}^{p \times q}$ is defined by
}

$$
A \otimes B=\left[\begin{array}{ccc}
a_{1,1} B & \ldots & a_{1, n} B \\
\vdots & \ddots & \vdots \\
a_{m, 1} B & \cdots & a_{m, n} B
\end{array}\right]
$$

being of size $m p \times n q$. For more information see e.g. Macedo \& Oliveira (2013). 
this result) and $e^{A \oplus B}=e^{A} \otimes e^{B}$. Eq. (8) can then be simplified by writing the vectorized version as

$$
\begin{aligned}
\operatorname{vec}\left(\operatorname{Var}\left[p(t) \mid \mathcal{F}_{s}^{B_{p}}\right]\right) & =\left(\int_{s}^{t} e^{-(t-u)(K \oplus K)} d u\right) \operatorname{vec}\left(\Sigma \Sigma^{\mathrm{T}}\right) \\
& =(K \oplus K)^{-1}\left(\mathbf{I}-e^{-(t-s) K \oplus K}\right) \operatorname{vec}\left(\Sigma \Sigma^{\mathrm{T}}\right) .
\end{aligned}
$$

Since we assume that the eigenvalues of $K$ all have positive real parts, all eigenvalues of $K \oplus K$ have positive real parts, see e.g. Laub (2005). Hence, the existence of a limiting distribution of $q(t)$ follows from Prop. 6.2 of Ichihara \& Kunita (1974). This limiting distribution is normal with mean zero and variance

$$
\lim _{t \rightarrow \infty} \operatorname{Var}\left[q(t) \mid \mathcal{F}_{s}^{B_{p}}\right]=\operatorname{vec}^{-1}\left((K \oplus K)^{-1} \operatorname{vec}\left(\Sigma \Sigma^{\mathrm{T}}\right)\right) .
$$

As time approaches infinity, the expected value of $p(t)$ will therefore coincide with $\Lambda(t)$, while the variance of $p(t)$ coincides with the variance of $q(t)$ given in Eq. (9).

\subsection{Properties of $z(t)$}

Limiting our investigation to the case $N=2$, we assume that the state of $I(t)$ is determined by a latent univariate stochastic process $z(t)$. Specifically, we assume that the EPC state occurs whenever $z(t)>0$, so in this two-dimensional case we have

$$
I(t)=\left\{\begin{array}{ll}
I_{(1)}, & \text { if } z(t)>0 \\
I_{(2)}, & \text { otherwise }
\end{array},\right.
$$

where $I_{(1)}$ and $I_{(2)}$ are given in Eq. (2). $z(t)$ is defined by

$$
\begin{aligned}
z(t) & =\lambda(t)+x(t), \\
\lambda(t) & =a_{\lambda}+b_{\lambda} \cos \left(\frac{2 \pi t}{M}+c_{\lambda}\right), \\
d x(t) & =-k x(t) d t+\sigma(t) d B_{z}(t), \\
\sigma(t) & =a_{\sigma}+b_{\sigma} \cos \left(\frac{2 \pi t}{M}+c_{\sigma}\right) .
\end{aligned}
$$

Here $a_{\lambda}, b_{\lambda}, c_{\lambda}, k, a_{\sigma}, b_{\sigma}, c_{\sigma}, M \in \mathbb{R}$ are constants, and $B_{z}(t)$ is a univariate Brownian motion. $\lambda(t)$ is a seasonal function measuring the asymptotic mean level of $z(t)$, while $x(t)$ measures the uncertainty of $z(t)$. To allow for time-varying uncertainty in $\mathbb{P}(z(t)>0)$, which is pronounced in the empirical investigations in Sec. 2 and especially Fig. 5, we introduce seasonality in the volatility parameter $\sigma(t)$.

We denote the filtration generated by the Brownian motion $B_{z}(t)$ by $\left\{\mathcal{F}_{t}^{B_{z}}\right\}_{t \geq 0}$, and assume independence between $\mathcal{F}_{t}^{B_{z}}$ and $\mathcal{F}_{t}^{B_{p}}$. Define $\mathcal{F}_{t}:=\mathcal{F}_{t}^{B_{z}} \vee \mathcal{F}_{t}^{B_{p}}$. The following proposition is useful in an estimation and simulation setting.

Proposition 2. Let $z(t)$ be given as in Eq. (10), and let $s<t$. Then the distribution of $z(t)$ is normal conditional on $\mathcal{F}_{s}$ with mean

$$
\mathbb{E}\left[z(t) \mid \mathcal{F}_{s}\right]=\lambda(t)+x(s) e^{-k(t-s)},
$$


and variance

$$
\operatorname{Var}\left[z(t) \mid \mathcal{F}_{s}\right]=\frac{a_{\sigma}^{2}}{2 k}\left(1-e^{-2 k(t-s)}\right)+A(s, t)+B(s, t)
$$

where

$$
\begin{gathered}
A(s, t)=b_{\sigma}^{2}\left(\frac{4 \pi^{2}+M^{2} k^{2}+M^{2} k^{2} \cos \left(\frac{4 \pi t}{M}+2 c_{\sigma}\right)+2 M k \pi \sin \left(\frac{4 \pi t}{M}+2 c_{\sigma}\right)}{4\left(M^{2} k^{3}+4 \pi^{2} k\right)}\right. \\
\left.-e^{-2 k(t-s)} \frac{4 \pi^{2}+M^{2} k^{2}+M^{2} k^{2} \cos \left(\frac{4 \pi s}{M}+2 c_{\sigma}\right)+2 M k \pi \sin \left(\frac{4 \pi s}{M}+2 c_{\sigma}\right)}{4\left(M^{2} k^{3}+4 \pi^{2} k\right)}\right), \\
B(s, t)=2 a_{\sigma} b_{\sigma} M\left(\frac{\pi \sin \left(\frac{2 \pi t}{M}+c_{\sigma}\right)+M k \cos \left(\frac{2 \pi t}{M}+c_{\sigma}\right)}{2\left(M^{2} k^{2}+\pi^{2}\right)}\right. \\
\left.-e^{-2 k(t-s)} \frac{\pi \sin \left(\frac{2 \pi s}{M}+c_{\sigma}\right)+M k \cos \left(\frac{2 \pi s}{M}+c_{\sigma}\right)}{2\left(M^{2} k^{2}+\pi^{2}\right)}\right) .
\end{gathered}
$$

Proof. Using the Ito formula, the solution to Eq. (11) for $s<t$ is

$$
x(t)=e^{-k(t-s)} x(s)+\int_{s}^{t} e^{-k(t-u)} \sigma(u) d B_{z}(u) .
$$

The normality follows from the deterministic integrand and the Brownian motion. The expected value is trivially calculated, and the variance is obtained by using the Ito isometry and straightforward calculations.

\subsubsection{An alternative approach}

One approach that might be obvious to use to model the underlying process governing the state of $I(t)$ is that of continuous-time Markov chains, having been shown to be of great usefulness in various applications (see e.g. Hubbard et al. (2008) in the context of disease progression modelling, and Inamura (2006) in the context of credit rating modelling). As already noted, the probability of EPC can be time-dependent with a seasonal pattern, see Fig. 5, meaning that a time-homogeneous Markov chain would fail to give a realistic representation of the underlying of $I(t)$. Time-inhomogeneous Markov chains would take care of the just-mentioned shortcoming, but, to the best of our knowledge, limited theoretical results exist to accommodate it. Hubbard et al. (2008) use a common time transformation to make transition rates time dependent, which is not applicable in our setting, since we would need a non-common time transformation to make certain states more likely than others in certain periods and vice versa. Time-inhomogeneous Markov chains being able to accommodate the above are therefore left as future research, which would possibly also pave the way for the case with $N>2$.

\subsection{Properties of $S(t)$}

Having established results regarding $p(t)$ and $z(t)$ in Sections 3.1 and 3.2, respectively, we now turn to $S(t)$. 
Proposition 3. Let $p(t)$ and $z(t)$ be defined as in Sections 3.1 and 3.2, respectively. The distribution of $S(t)$ is then mixed normal conditional on $\mathcal{F}_{s}$ for $s<t$ with density

$$
f_{s, t}(x)=\sum_{j=1}^{2} \mathbb{P}_{(j)}(s, t) \frac{1}{(2 \pi)^{N_{j} / 2}\left|\Sigma_{(j)}(s, t)\right|} e^{-\frac{1}{2}\left(x-\mu_{(j)}(s, t)\right)^{\mathrm{T}} \Sigma_{(j)}(s, t)^{-1}\left(x-\mu_{(j)}(s, t)\right)}
$$

with $N_{j} \leq N$ for $j=\{1,2\}$ being the rank of $I_{(j)}, \Phi(\cdot)$ being the cumulative distribution function for the standard normal distribution, and where

$$
\begin{aligned}
\mu_{(j)}(s, t) & =I_{(j)} \mathbb{E}\left[p(t) \mid \mathcal{F}_{s}\right] \\
\Sigma_{(j)}(s, t) & =I_{(j)} \mathbb{V} \operatorname{ar}\left[p(t) \mid \mathcal{F}_{s}\right] I_{(j)}^{\mathrm{T}},
\end{aligned}
$$

and

$$
\mathbb{P}_{(1)}(s, t)=1-\Phi\left(\frac{-\mathbb{E}\left[z(t) \mid \mathcal{F}_{s}\right]}{\sqrt{\operatorname{Var}\left[z(t) \mid \mathcal{F}_{s}\right]}}\right), \quad \mathbb{P}_{(2)}(s, t)=1-\mathbb{P}_{(1)}(s, t)
$$

Proof. The characteristic function of $S(t)$ is

$$
\begin{aligned}
& \mathbb{E}\left[e^{i y^{\mathrm{T}} S(t)} \mid \mathcal{F}_{S}\right]=\mathbb{E}\left[e^{i y^{\mathrm{T}} I(t)(\Lambda(t)+q(t))} \mid \mathcal{F}_{s}\right] \\
& =\mathbb{E}\left[\mathbb { E } \left[\operatorname { e x p } \left(i y ^ { \mathrm { T } } I ( t ) \left(\Lambda(t)+e^{-(t-s) K} q(s)\right.\right.\right.\right. \\
& \left.\left.\left.\left.+\int_{s}^{t} e^{-(t-u) K} \Sigma d B_{p}(u)\right)\right) \mid \mathcal{F}_{t}^{B_{z}} \vee \mathcal{F}_{s}\right] \mid \mathcal{F}_{s}\right] \\
& =\mathbb{E}\left[\operatorname { e x p } \left(i y^{\mathrm{T}} I(t)\left(\Lambda(t)+e^{-(t-s) K} q(s)\right)\right.\right. \\
& \left.\left.-\frac{1}{2} y^{\mathrm{T}} I(t) \int_{s}^{t} e^{-(t-u) K} \Sigma \Sigma^{\mathrm{T}} e^{-(t-u) K^{\mathrm{T}}} d u I(t)^{\mathrm{T}} y\right) \mid \mathcal{F}_{s}\right] \\
& =\mathbb{P}\left(z(t)>0 \mid \mathcal{F}_{s}\right) \exp \left(i y^{\mathrm{T}} I_{(1)}\left(\Lambda(t)+e^{-(t-s) K} q(s)\right)\right. \\
& \left.-\frac{1}{2} y^{\mathrm{T}} I_{(1)} \int_{s}^{t} e^{-(t-u) K} \Sigma \Sigma^{\mathrm{T}} e^{-(t-u) K^{\mathrm{T}}} d u I_{(1)}^{\mathrm{T}} y\right) \\
& +\left(1-\mathbb{P}\left(z(t)>0 \mid \mathcal{F}_{s}\right)\right) \exp \left(i y^{\mathrm{T}} I_{(2)}\left(\Lambda(t)+e^{-(t-s) K} q(s)\right)\right. \\
& \left.-\frac{1}{2} y^{\mathrm{T}} I_{(2)} \int_{s}^{t} e^{-(t-u) K} \Sigma \Sigma^{\mathrm{T}} e^{-(t-u) K^{\mathrm{T}}} d u I_{(2)}^{\mathrm{T}} y\right) .
\end{aligned}
$$

The second equality is a consequence of the law of iterated expectations. As a result of Prop. 2, we further have that

$$
\mathbb{P}\left(z(t)>0 \mid \mathcal{F}_{s}\right)=1-\Phi\left(\frac{-\mathbb{E}\left[z(t) \mid \mathcal{F}_{s}\right]}{\sqrt{\operatorname{Var}\left[z(t) \mid \mathcal{F}_{s}\right]}}\right)
$$

concluding the proof. 
The construction of the model for $S(t)$ implies that the introduced seasonal time variation in $p(t)$ and $z(t)$ translates to seasonal time variation in $S(t)$. To briefly elaborate on this, observe that the expected value of $S(t)$ is

$$
\begin{aligned}
\mathbb{E}\left[S(t) \mid \mathcal{F}_{s}\right] & =\mathbb{E}\left[I(t) \mathbb{E}\left[p(t) \mid \mathcal{F}_{t}^{B_{z}} \vee \mathcal{F}_{s}\right] \mid \mathcal{F}_{s}\right] \\
& =\left[\mathbb{P}_{(1)}(s, t) I_{(1)}+\left(1-\mathbb{P}_{(1)}(s, t)\right) I_{(2)}\right] \mathbb{E}\left[p(t) \mid \mathcal{F}_{s}\right]
\end{aligned}
$$

Since we in both $\mathbb{P}_{(1)}(s, t)$ and $\mathbb{E}\left[p(t) \mid \mathcal{F}_{s}\right]$ can allow seasonal time variation, the expected value of $S(t)$ will thus be affected by these specifications. Eq. (18) has a very intuitive interpretation: The expected value of day-ahead prices is a weighting of 1) the expected value of day-ahead prices if EPC is present, and 2) the expected domestic prices. The weights are given by the probability of being in each state.

\section{Estimation}

Due to the assumed independence between $\mathcal{F}_{t}^{B_{z}}$ and $\mathcal{F}_{t}^{B_{p}}$, the estimation procedure can be divided in two independent parts. Namely, estimation of the parameters governing $p(t)$, and estimation of the parameters governing $z(t)$. A common feature is the latent behaviour of the considered processes. We therefore use filtering techniques in both cases.

Besides these two components, we also consider estimation of the weights constituting $I(t)$ inspired by spatial econometrics, which turns out to be estimation of $w$ in our two-market case, cf. Eq. (2). We consider each of them in turn in the following sections.

\subsection{Estimation of $p(t)$}

We assume that we have an equidistant sampling between observations and mark it by $\Delta$. Eqs. (7)-(8) implies that the state equation of $q(t)$ in a state space representation is

$$
q(t)=F(t) q(t-\Delta)+\eta(t)
$$

where $F(t)=e^{-\Delta K}, \eta(t) \sim \mathrm{N}\left(0, Q_{t}\right)$, and $Q_{t}=\operatorname{Var}\left[q(t) \mid \mathcal{F}_{t-\Delta}\right]$. Given the estimation purpose of this section, we introduce a measurement error in Eq. (1) and define the measurement equation by

$$
\begin{aligned}
S(t) & =I(t) p(t)+\epsilon(t) \\
& =I(t)(\Lambda(t)+q(t))+\epsilon(t),
\end{aligned}
$$

where $\epsilon(t) \sim \mathrm{N}(0, R(t)) . R(t)$ is

$$
R(t)=\left\{\begin{array}{ll}
R_{(1)}, & \text { if } I(t)=I_{(1)} \\
R_{(2)}, & \text { if } I(t)=I_{(2)}
\end{array},\right.
$$

with $R_{(1)}$ and $R_{(2)}$ being constant diagonal matrices corresponding to the EPC and non-EPC state, respectively.

\subsubsection{Kalman filtering}

The normality and linearity of the state space representation lead us to using the Kalman filter in combination with maximum likelihood to estimate the parameters of $p(t)$. There is vast literature on the Kalman filter; see e.g. Schwartz \& Smith (2000) in the context of commodity markets. 
During the predict step of the Kalman filtering procedure, the a priori estimate of the state is

$$
\hat{q}(t \mid t-\Delta)=F(t) \hat{q}(t-\Delta \mid t-\Delta),
$$

and the a priori estimate of covariance of this state estimate is

$$
\hat{P}(t \mid t-\Delta)=F(t) \hat{P}(t-\Delta \mid t-\Delta) F(t)^{T}+Q(t) .
$$

We update the state estimate and the corresponding covariance by

$$
\hat{q}(t \mid t)=\hat{q}(t \mid t-\Delta)+H(t)(S(t)-I(t)(\Lambda(t)+\hat{q}(t \mid t-\Delta))),
$$

and

$$
\hat{P}(t \mid t)=\hat{P}(t \mid t-\Delta)-H(t) I(t) \hat{P}(t \mid t-\Delta),
$$

respectively, where

$$
H(t)=\hat{P}(t \mid t-\Delta) I(t)^{T}\left(I(t) \hat{P}(t \mid t-\Delta) I(t)^{T}+R(t)\right)^{-1}
$$

is the Kalman gain. Due to the assumed independence between $\mathcal{F}_{t}^{B_{z}}$ and $\mathcal{F}_{t}^{B_{p}}$, we regard $I(t)$ as given at time $t$ in the Kalman filtering scheme.

Let $T$ denote the number of observations, and let $\theta_{\Lambda}$ denote the parameters governing the deterministic function $\Lambda(t)$. Having established the Kalman filtering scheme, we maximize the log-likelihood

$$
\mathcal{L}\left(\theta_{q}\right)=\sum_{i=2}^{T} \log f\left(S(i) \mid \mathcal{F}_{i}^{B_{z}}, \mathcal{F}_{i-1}^{B_{p}}\right),
$$

with respect to $\theta_{q}=\left(\theta_{\Lambda}, K, \Sigma, R_{(1)}, R_{(2)}\right)$, and where

$$
\begin{gathered}
f\left(S(i) \mid \mathcal{F}_{i}^{B_{z}}, \mathcal{F}_{i-1}^{B_{p}}\right)=|2 \pi V(i)|^{-1 / 2} e^{-\frac{1}{2}(S(i)-E(i))^{T} V(i)^{-1}(S(i)-E(i))}, \\
E(i)=I(i)(\Lambda(i)+\hat{q}(i \mid i-1)),
\end{gathered}
$$

and

$$
V(i)=I(i) \hat{P}(i \mid i-1) I(i)^{T}+R(i) .
$$

\subsection{Estimation of $z(t)$}

Let $y(t) \in\{0,1\}$ denote the observed state of $I(t)$ at time $t$, meaning that $y(t)$ is a Bernoulli process with dependent and non-identically distributed Bernoulli trials. To see this, we assume that $y(t)=1$ corresponds to EPC. Therefore, by remembering Eq. (17) and Prop. 2, we get

$$
\begin{aligned}
\mathbb{P}\left(y(t)=1 \mid \mathcal{F}_{s}\right) & =\mathbb{P}\left(z(t)>0 \mid \mathcal{F}_{s}\right) \\
& =1-\Phi\left(\frac{-\mathbb{E}\left[z(t) \mid \mathcal{F}_{s}\right]}{\sqrt{\operatorname{Var}\left[z(t) \mid \mathcal{F}_{s}\right]}}\right) \\
& =1-\Phi\left(\frac{-\left(\lambda(t)+e^{-k(t-s)} x(s)\right)}{\sqrt{\operatorname{Var}\left[z(t) \mid \mathcal{F}_{s}\right]}}\right) .
\end{aligned}
$$


Hence, due to the dependence on $x(s)$ we have that the Bernoulli trials are dependent and non-identically distributed.

As a result of Eq. (20), the conditional density of $y(t)$ is

$$
f\left(y(t) \mid \mathcal{F}_{s}\right)=\left(1-\Phi\left(\frac{-\left(\lambda(t)+e^{-k(t-s)} x(s)\right)}{\sqrt{\mathbb{V a r}\left[z(t) \mid \mathcal{F}_{s}\right]}}\right)\right)^{y(t)} \Phi\left(\frac{-\left(\lambda(t)+e^{-k(t-s)} x(s)\right)}{\sqrt{\operatorname{Var}\left[z(t) \mid \mathcal{F}_{s}\right]}}\right)^{1-y(t)} .
$$

Standard estimation methods such as maximum likelihood estimation is not feasible, since the log-likelihood,

$$
\mathcal{L}\left(\theta_{z}\right)=\sum_{i=2}^{T} \log \left(1-\Phi\left(\frac{-\left(\lambda(i)+e^{-k \Delta} x(i-1)\right)}{\sqrt{\operatorname{Var}\left[z(i) \mid \mathcal{F}_{i-1}\right]}}\right)\right)^{y(i)} \Phi\left(\frac{-\left(\lambda(i)+e^{-k \Delta} x(i-1)\right)}{\sqrt{\operatorname{Var}\left[z(i) \mid \mathcal{F}_{i-1}\right]}}\right)^{1-y(i)}
$$

where $\theta_{z}=\left(a_{\lambda}, b_{\lambda}, c_{\lambda}, k, a_{\sigma}, b_{\sigma}, c_{\sigma}\right)$ cf. Sec. 3.2, depends on the latent process $x(t)$. The linear Kalman filter is suitable in the estimation of $p(t)$, but the non-differentiable relationship between $y(t)$ and $z(t)$ additionally invalidates the extended Kalman filter, and we are thus in the need for another approach.

\subsubsection{Particle filtering}

We choose to use particle filtering to mitigate this, and specifically we have employed the bootstrap filter. For references, the interested reader is advised to look at Kantas et al. (2015) in a general state-space model, whereas Pitt et al. (2014) consider particle filtering in the context of stochastic volatility models. As in the Kalman filter case, the bootstrap filter can be divided in two steps: a predict and an update step $^{\dagger}$. In our context, the bootstrap procedure is as follows:

Let again $T$ denote the number of observations. For $t=\Delta, 2 \Delta, \ldots, T-\Delta$ we do the following:

- Predict step

1. Draw $n$ samples $\hat{x}_{i}(t \mid t-\Delta) \sim \mathrm{N}\left(e^{-k \Delta} \hat{x}_{i}(t-\Delta \mid t-\Delta), \operatorname{Var}\left[x(t) \mid \mathcal{F}_{t-\Delta}\right]\right)$, cf. Prop. 2.

- Update step

2. For each $i \in\{1,2, \ldots, n\}$, calculate weights

$$
\begin{aligned}
\gamma_{i}(t+\Delta)= & \left(1-\Phi\left(\frac{-\left(\lambda(t+\Delta)+e^{-k \Delta} \hat{x}_{i}(t \mid t-\Delta)\right)}{\sqrt{\operatorname{Var}\left[z(t+\Delta) \mid \mathcal{F}_{t}\right]}}\right)\right)^{y(t+\Delta)} \\
& \times \Phi\left(\frac{-\left(\lambda(t+\Delta)+e^{-k \Delta} \hat{x}_{i}(t \mid t-\Delta)\right)}{\sqrt{\operatorname{Var}\left[z(t+\Delta) \mid \mathcal{F}_{t}\right]}}\right)^{1-y(t+\Delta)}
\end{aligned}
$$

cf. Eq. (21).

3. For each $i \in\{1,2, \ldots, n\}$, calculate normalized weights,

$$
\tilde{\gamma}_{i}(t+\Delta)=\frac{\gamma_{i}(t+\Delta)}{\sum_{j=1}^{M} \gamma_{j}(t+\Delta)}
$$

${ }^{\dagger}$ The bootstrap filter can also be divided in an importance and selection step, but we find it more intuitive to divide the algorithm in a predict and update step, also making the Kalman filter presented earlier and the bootstrap filter easily comparable. 
4. For each $i \in\{1,2, \ldots, n\}$, draw a sample $\hat{x}_{i}(t \mid t)$ from the distribution with density

$$
f(x)=\sum_{j=1}^{n} \tilde{\gamma}_{j}(t+\Delta) \delta\left(x-\hat{x}_{j}(t \mid t-\Delta)\right),
$$

where $\delta(\cdot)$ is Dirac's delta function.

Having obtained $n$ values of $x(t)$ (i.e., $z(t))$ for each $t=0, \Delta, \ldots, T-\Delta$, we evaluate the $\log$ likelihood as

$$
\begin{aligned}
\mathcal{L}\left(\theta_{z}\right)=\sum_{i=2}^{T} \log \left(\frac{1}{n} \sum_{j=1}^{n}\right. & \left(1-\Phi\left(\frac{-\left(\lambda(i)+e^{-k \Delta} \hat{x}_{j}(i-1 \mid i-2)\right)}{\sqrt{\operatorname{Var}\left[z(i) \mid \mathcal{F}_{i-1}\right]}}\right)\right)^{y(i)} \\
\times & \left.\left(\frac{-\left(\lambda(i)+e^{-k \Delta} \hat{x}_{j}(i-1 \mid i-2)\right)}{\sqrt{\operatorname{Var}\left[z(i) \mid \mathcal{F}_{i-1}\right]}}\right)^{1-y(i)}\right) .
\end{aligned}
$$

As pointed out by Pitt et al. (2014), one single run of the particle filter will therefore estimate the log-likelihood.

Due to the non-observable nature of the $z(t)$ process and the fact that the sign of it only matters, one has to fix the level of it in order to avoid possible oscillating estimation issues. Hence, we fix $\sigma(t)$ to oscillate around 1 , by setting $a_{\sigma}$ to

$$
a_{\sigma}=\sqrt{\frac{2 k}{1-e^{-2 k \Delta}}} .
$$

\subsection{Estimation of $I(t)$}

Inspired by weight matrix determination in spatial econometrics (see e.g. Abate \& Haldrup (2017)), we construct $I(t)$ using the spatial relationship between the market areas. Although our model differs from spatial econometric models in that the observed prices do not depend on lagged values of observed prices from the market areas under consideration, but rather depend on a weighting of (partly) unobservable domestic prices, the weighting of the unobservable domestic prices are chosen in a similar way. As discussed in Abate \& Haldrup (2017), choosing the spatial weight matrix is a delicate task, due to the selection of the factor influencing the weight matrix. Since net export basically results in a horizontal shift in either the domestic supply or demand curve, depending on the sign of the net export cf. Fig. 1, the consumed electricity in each market area determines how sensitive the resulting domestic market price is to variation in net export. Loosely speaking, a large market area - based on the supplied electricity — is not as price sensitive to net export as a smaller market area. Hence, we use the supplied electricity in a market area as the "size" of the market area. In the two-market case, estimation of $I(t)$ boils down to estimating $w$, cf. Eq. (2).

\section{Application}

In this section, we first exemplify the proposed model by jointly modelling hour 21 day-ahead prices for Germany and France. Presentation of estimation results is then followed by an analysis of derivative prices implied by the model. 


\subsection{Case study of German and French electricity prices}

Our data set introduced in Sec. 2 contains daily observations. Hence, we set $M=365$, and the time between adjacent observations is set to $1 / 365$. We report estimation results for $p(t)$ and $z(t)$ in the following sections after a short note on the estimation of $w$ in $I_{(1)}$. As discussed in Sec. 4.3, we use the supplied electricity to determine $w$. Table 2 reports the supplied electricity for

\begin{tabular}{cc}
\hline DE & FR \\
\hline 575,701 & 496,932 \\
\hline
\end{tabular}

Table 2: Supplied electricity given in (GWh) based on IEA (2017).

Germany and France, which results in a weight for Germany of 575, 701/(575, $701+496,932)=$ 0.537. The resulting two states of $I(t)$ are given in Eq. (22).

$$
I_{(1)}=\left[\begin{array}{ll}
0.537 & 0.463 \\
0.537 & 0.463
\end{array}\right] \text { or } I_{(2)}=\left[\begin{array}{ll}
1 & 0 \\
0 & 1
\end{array}\right] \text {. }
$$

Motivated by the empirical findings in Sec. 2 and related literature on electricity price modeling (e.g. Pircalabu \& Benth (2017)), we define the seasonal function $\Lambda(t)$ in Eq. (3) as

$$
\Lambda(t)=a_{\Lambda}+b_{\Lambda} \cos \left(\frac{2 \pi t}{M}\right)+c_{\Lambda} \sin \left(\frac{2 \pi t}{M}\right)+d_{\Lambda} I_{W}(t)
$$

where $I_{W}(t)$ is an indicator function being one if day $t$ is either Saturday or Sunday, and $a_{\Lambda}, b_{\Lambda}, c_{\Lambda}, d_{\Lambda} \in \mathbb{R}^{2}$ are constants. We further assume that $\Sigma$ is lower triangular, and that $K$ is diagonal in Eq. (4). In Tables 3-4 the estimation results for $\theta_{q}$ are reported using the estimation method presented in Sec. 4. To simplify the presentation, the estimation results are divided in the parameters governing $\Lambda(t)$ and $q(t)$.

\begin{tabular}{ccccc}
\hline & $\hat{a}_{\Lambda}$ & $\hat{b}_{\Lambda}$ & $\hat{c}_{\Lambda}$ & $\hat{d}_{\Lambda}$ \\
\hline $\mathrm{DE}$ & 36.42 & 0.75 & 3.43 & -4.74 \\
\hline $\mathrm{FR}$ & 45.17 & 4.52 & -7.65 & -6.34 \\
\hline
\end{tabular}

Table 3: Estimation results for $\Lambda(t)$.

\begin{tabular}{lllll}
\hline$\hat{K}_{(1,1)}$ & $\hat{K}_{(2,2)}$ & $\hat{\Sigma}_{(1,1)}$ & $\hat{\Sigma}_{(2,1)}$ & $\hat{\Sigma}_{(2,2)}$ \\
\hline 89.53 & 30.68 & 99.95 & 52.34 & 52.15 \\
\hline
\end{tabular}

Table 4: Estimation results for $q(t)$.

The estimated weekend coefficient, $\hat{d}_{\Lambda}$ for both market areas is negative, and thus reflects the lower electricity demand during weekends compared to weekdays. Also notable is the estimated base level $\hat{a}_{\Lambda}$, where the German base level is lower than the French base level. Regarding the speed of mean reversion, the estimated German one, $\hat{K}_{(1,1)}$, corresponds to a half-life of approximately 2.8 days, whereas the estimated French speed of mean reversion corresponds to a more persistent behaviour with a half-life of approximately 8.2 days. 


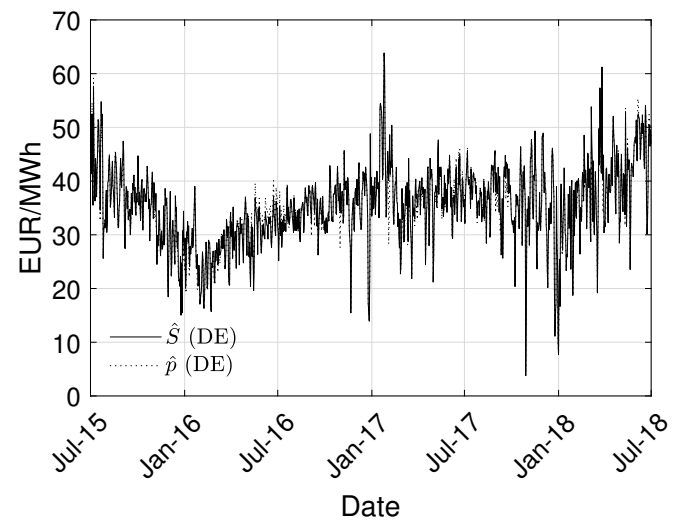

(a) $\hat{S}$ and $\hat{p}$ for Germany

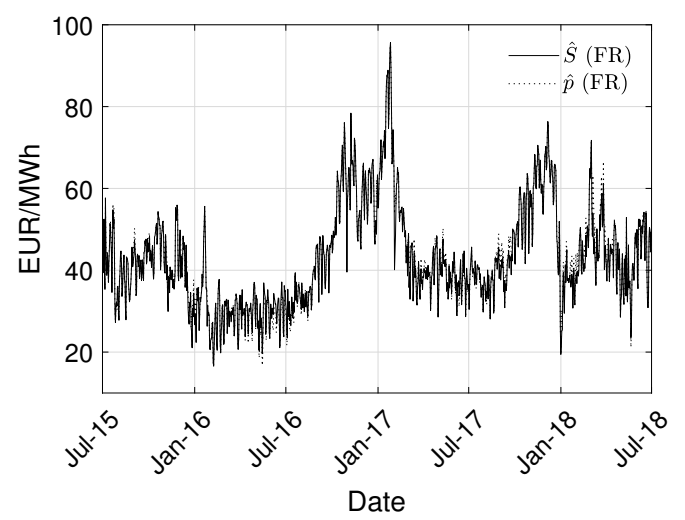

(c) $\hat{S}$ and $\hat{p}$ for France

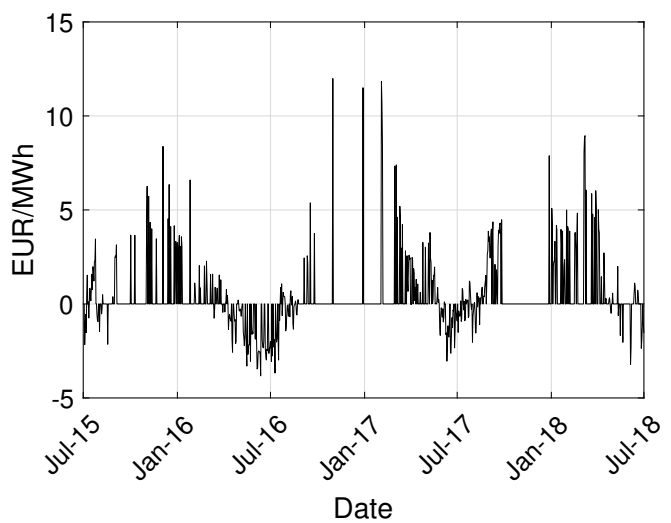

(b) $\hat{S}$ - $\hat{p}$ for Germany

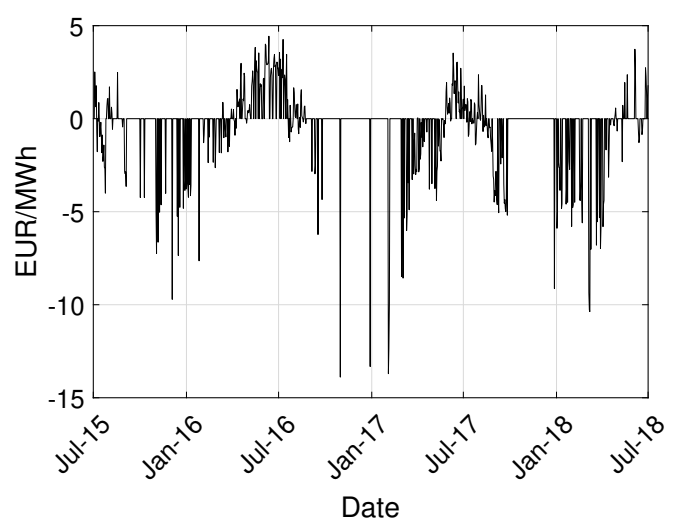

(d) $\hat{S}-\hat{p}$ for France

Figure 6: (a) Estimated $\hat{S}$ and $\hat{p}$ for Germany, (c) $\hat{S}-\hat{p}$ for Germany, (c) estimated $\hat{S}$ and $\hat{p}$ for France, (d) $\hat{S}-\hat{p}$ for France.

To investigate the estimation results in more detail, we obtain the estimated $\hat{p}$ process by filtering of $\hat{q}$, following the Kalman scheme described in Sec. 4. In addition, using the estimated forms of $I(t)$ presented in Eq. (22), and assuming we have knowledge of $I(t)$ at time $t$ as in the described Kalman scheme, we also obtain the estimated $\hat{S}$ process. The intention of showing the estimated $\hat{S}$ process in addition to the estimated $\hat{p}$ process is twofold. Firstly, to give a sense of the model's ability to replicate the observed prices in Fig. 3(a), and secondly to give an insight in the difference between the model implied $\hat{S}$ and $\hat{p}$ processes. In Figs. 6(a) and 6(c) we show the estimated $\hat{p}$ process and the corresponding estimated $\hat{S}$ process for both Germany and France, resembling the observations in Fig. 3(a) quite well. Figs. 6(b) and 6(d) illustrate the difference between the estimated $\hat{S}$ and $\hat{p}$ processes. It is apparent that the differences are more frequent during spring/summer than autumn/winter, reflecting the fact that EPC happens more frequently during spring/summer. Also notable is the fact that the differences are greater during autumn/winter, so from this perspective, the impact from EPC on a single observation is highest during autumn/winter. Due to the parameter $w$ affecting the weighting of the domestic day-ahead prices (the $p$ process), the resulting differences between the $\hat{S}$ and $\hat{p}$ processes in the two market areas differ. The pattern in Fig. 6(b) is therefore reversed in Fig. 6(d), but they differ in absolute terms. 
The estimated parameters for the $z(t)$ process are reported in Table 5. The estimated speed of mean reversion translates to a half-life of approximately 2.56 days. Fig. $7($ a) shows the es-

\begin{tabular}{lccccc}
\hline$\hat{k}$ & $\hat{a}_{\lambda}$ & $\hat{b}_{\lambda}$ & $\hat{c}_{\lambda}$ & $\hat{b}_{\sigma}$ & $\hat{c}_{\sigma}$ \\
\hline 98.8570 & -0.6146 & 1.3094 & 0.0565 & -14.2477 & -1.3537 \\
\hline
\end{tabular}

Table 5: Estimation results for $z(t)$.

timated asymptotic probability of observing EPC throughout the year. The yearly pattern observed in Fig. 5(a) is thus replicated by the model. The significant drop in probability during August/September is notable and marks the switch from summer to autumn. The lowest probability is reached in November and is as low as $0.2 \%$, while the highest is in July and is $82 \%$. In a risk management or asset valuation situation, this is quite a difference and must be taken into account. We elaborate on this matter when covering derivative prices implied by the model.

Remember that we assume independence between $\mathcal{F}_{t}^{B_{z}}$ and $\mathcal{F}_{t}^{B_{p}}$, which implies that we can divide the estimation procedure in two independent parts. Fig. 7 (c) shows a histogram of the observed price spread between France and Germany. To investigate the dependence between the $p(t)$ process and the $z(t)$ process, we only show price spreads from non-EPC days, which is immediately followed by days with EPC. Most occurrences are observed just about zero, meaning that there is a tendency that EPC happens in periods with small price spreads. Though, there is no guarantee that EPC only happens in these periods, since it reaches as high as approximately $27 \mathrm{EUR} / \mathrm{MWh}$ in absolute terms. From the perspective of the estimated model, Fig. 7(b) shows the difference between the asymptotic mean levels of $p(t)$; i.e., it shows the spread between $\Lambda_{1}(t)$ and $\Lambda_{2}(t)$, where $\Lambda(t)=\left[\begin{array}{ll}\Lambda_{1}(t) & \Lambda_{2}(t)\end{array}\right]^{\top}$. Comparing the shape of Fig. $7(\mathrm{~b})$ to the shape of Fig. 7(a), we conclude that a high probability of EPC roughly corresponds to a small price spread asymptotically, and vice versa. Loosely speaking, even though we assume independence between $\mathcal{F}_{t}^{B_{z}}$ and $\mathcal{F}_{t}^{B_{p}}$, the seasonal components of the model suggest that a high probability of EPC translates to a small spread between the components of $p(t)$, and that a low probability of EPC translates to a large spread between the components of $p(t)$. Assuming independence between $\mathcal{F}_{t}^{B_{z}}$ and $\mathcal{F}_{t}^{B_{p}}$ thus has the advantage that we can split up the estimation procedure in two independent parts, while still allowing the above discussed dependency between the EPC probability and the magnitude of the spread between the components of $p(t)$. We leave it as future work to investigate the case with dependence between $\mathcal{F}_{t}^{B_{z}}$ and $\mathcal{F}_{t}^{B_{p}}$.

To further illustrate the model, we show in Fig. 8(a) a simulated path for both the German and French prices using the parameters reported in Tables 3-5. Again we emphasize when EPC occurs by a solid black line. As expected from Fig. 7(a) we observe most EPC states during summer, and least during autumn/winter. Regarding the EPC aspect of the model, we plot in Fig. $8(\mathrm{~b})$ the simulated $z(t)$ process, which is, due to the construction, positive whenever we observe EPC in Fig. 8(a).

\subsection{Derivative pricing}

In electricity markets, the underlying cannot be stored - at least not effectively - and the classical pricing assumptions about market completeness do therefore not hold. Hence, one typically resorts to imposing a parametric form through a measure change, and thereby introducing the so-called market price of risk. By using market data, it is then possible to back out the market price of risk. 


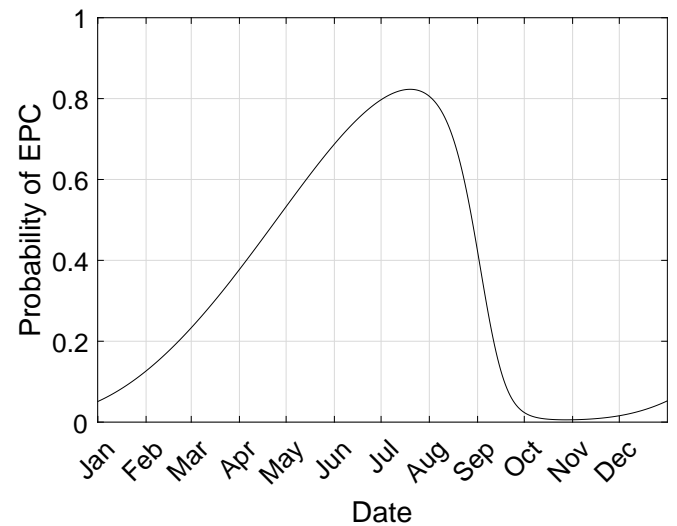

(a) Asymptotic probability of EPC.

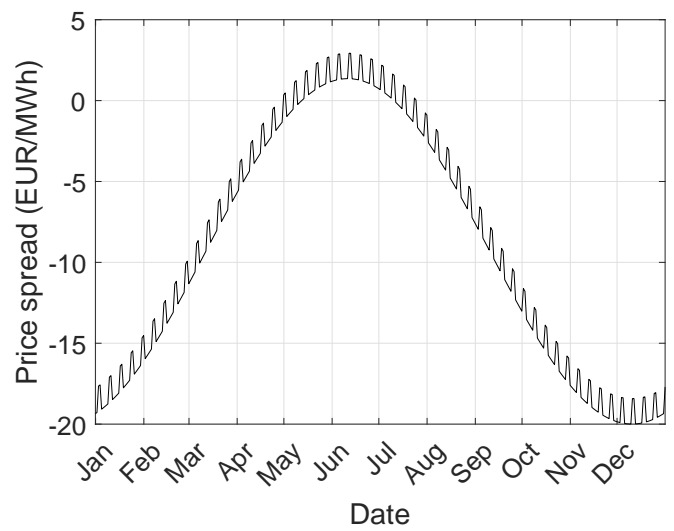

(b) Difference between the seasonality functions.

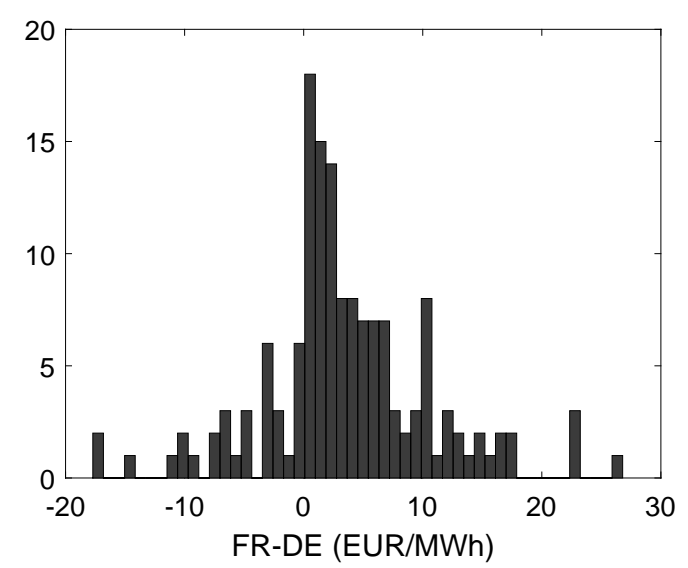

(c) FR-DE day-ahead price spread.

Figure 7: (a) Estimated asymptotic probability of observing EPC throughout a year, (b) difference between the estimated seasonality functions affecting the $p$ process, and (c) the observed German hour 21 day-ahead price subtracted from the French hour 21 day-ahead price. The prices used in (c) are prices from non-EPC days, where we observe EPC the next day. 


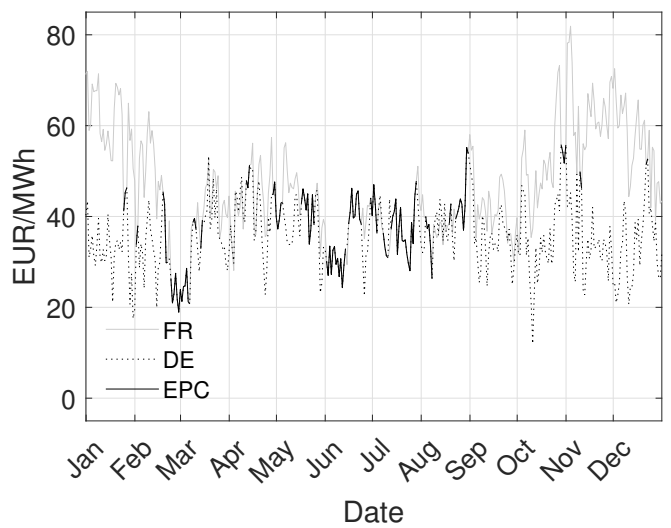

(a) Simulated DE and FR prices

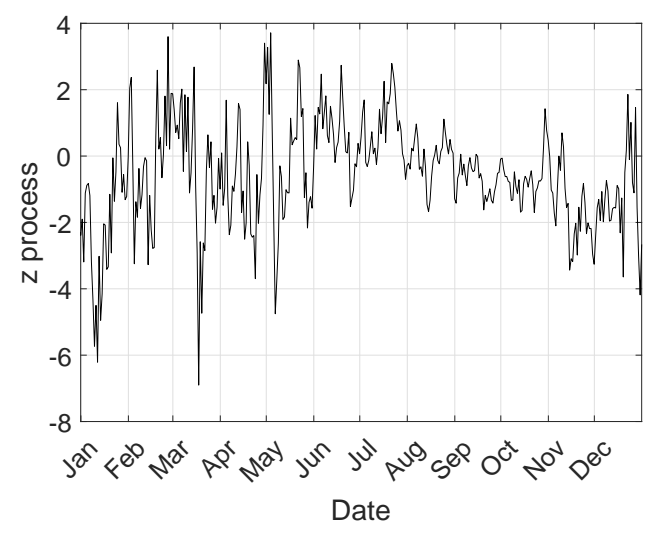

(b) Simulated $z$ process

Figure 8: (a) Simulated hour 21 day-ahead prices for Germany and France, and (b) corresponding simulation of $z$ process.

The Girsanov theorem gives the $p$-process under an equivalent pricing measure $Q$ as

$$
p(t)=\Lambda(t)+e^{-(t-s) K} q(s)+\int_{s}^{t} e^{-(t-u) K} \Sigma \varphi(u) d u+\int_{s}^{t} e^{-(t-u) K} \Sigma d B_{p}^{Q}(u) .
$$

where $\varphi(t) \in \mathbb{R}^{N}$ is the so-called market price of risk, assumed to be adapted to $\left\{\mathcal{F}_{t}\right\}_{t \geq 0}$ and satisfying certain integrability conditions (see Girsanov (1960)). In addition, $B_{p}^{Q}(t) \in \mathbb{R}^{N}$ is a Brownian motion under the new measure $Q$. By assuming no market price of risk concerning $z(t)$, we thus see that an appropriate shift in the mean level of $p(t)$ will yield the appropriate measure change.

In practice, one might assume that $\varphi(t)$ is a piecewise constant deterministic function and match it to the observed forward prices at time $s$, which we will cover in the next section. Eq. (23) then implies that $p(t)$ under $Q$ is normally distributed with expected value

$$
\mathbb{E}^{Q}\left[p(t) \mid \mathcal{F}_{s}\right]=\Lambda(t)+e^{-(t-s) K} q(s)+\int_{s}^{t} e^{-(t-u) K} \Sigma \varphi(u) d u
$$

and variance given in Eq. (6). It follows from Eq. (24) and Prop. 3 that $S(t)$ under $Q$ is characterized by the density

$$
f_{s, t}^{Q}(x)=\sum_{i=1}^{2} \mathbb{P}_{(i)}(s, t) \frac{1}{(2 \pi)^{N_{i} / 2}\left|\Sigma_{(i)}(s, t)\right|} e^{-\frac{1}{2}\left(x-\mu_{(i)}^{Q}(s, t)\right)^{\mathrm{T}} \Sigma_{(i)}(s, t)^{-1}\left(x-\mu_{(i)}^{Q}(s, t)\right)},
$$

where

$$
\mu_{(i)}^{Q}(s, t)=I_{(i)} \mathbb{E}^{Q}\left[p(t) \mid \mathcal{F}_{s}\right],
$$

and everything else is defined in Prop. 3.

\subsubsection{Forwards}

The liquidity of electricity markets is low compared to traditional financial markets, with the forward market in general being the most liquid. As a result, derivative prices are often restricted to be consistent with the forward market ${ }^{\dagger}$, which is used to back out the market price of risk.

\footnotetext{
${ }^{\dagger}$ We assume a deterministic interest rate, and thus the term forward market does also cover the futures market.
} 
Following the lines of the discussion of market incompleteness in electricity markets, we define the forward price at time $s$ for a forward contract with delivery time $t$ as the expected value under $Q$ of the future day-ahead price, as in e.g. Benth \& Meyer-Brandis (2009). In terms of our multivariate model, we have

$$
F(s, t)=\mathbb{E}^{Q}\left[S(t) \mid \mathcal{F}_{s}\right]
$$

where $F(s, t) \in \mathbb{R}^{N}$ is the forward price at time $s$ for delivery at time $t$ for $N$ different market areas. In our modeling framework, Prop. 4 states the forward price.

Proposition 4. Let $S(t)$ be defined under $Q$ as in Eq. (25). Then the forward price at time $s$ for delivery at time $t$ for two different market areas is

$$
F(s, t)=\left[\mathbb{P}_{(1)}(s, t) I_{(1)}+\mathbb{P}_{(2)}(s, t) I_{(2)}\right] \mathbb{E}^{Q}\left[p(t) \mid \mathcal{F}_{s}\right]
$$

Proof. Follows from Eq. (18) and Eq. (25).

Eq. (27) implies that the spread between forward prices decreases gradually as $\mathbb{P}_{(1)}(s, t) \rightarrow 1$. In the extreme case where $\mathbb{P}_{(1)}(s, t)=1$, the model suggest that the forward prices in the two different market areas are equal. The model is hence in line with the expectation of a single forward price in a situation with two perfectly coupled markets.

\subsubsection{Transmission rights}

A transmission right is a European spread option with the underlying assets being day-ahead prices of adjacent market areas. The pricing formula for transmission rights given the model presented in Sec. 3 is closely related to the work by Bachelier (1900) and is given in the next proposition.

Proposition 5. Let $S(t)$ be defined under $Q$ as in Eq. $(25), g \in \mathbb{R}^{2}$, and let $\left(g^{\mathrm{T}} s(t)-H\right)^{+}$be the payoff of a European spread option with strike $H$. Then

$$
\mathbb{E}^{Q}\left[\left(g^{\mathrm{T}} S(t)-H\right)^{+} \mid \mathcal{F}_{s}\right]=\sum_{i=1}^{2} \mathbb{P}_{(i)}(s, t)\left(a_{(i)}(s, t) \Phi\left(\frac{a_{(i)}(s, t)}{c_{(i)}(s, t)}\right)+c_{(i)}(s, t) \phi\left(\frac{a_{(i)}(s, t)}{c_{(i)}(s, t)}\right)\right),
$$

where

$$
\begin{aligned}
& a_{i}(s, t)=g^{\mathrm{T}} \mu_{(i)}^{Q}(s, t)-H, \\
& c_{i}(s, t)=\sqrt{g^{\mathrm{T}} \Sigma_{(i)}(s, t) g},
\end{aligned}
$$

and $\Phi$ and $\phi$ is the distribution and density function of the standard normal distribution, respectively. $\mu_{(i)}^{Q}(s, t)$ is given in Eq. (26), and $\Sigma_{(i)}(s, t)$ is given in Prop. 3.

Proof. Define $Z(t):=g^{\mathrm{T}} S(t)-H$. Eq. (25) implies that $Z(t)$ under $Q$ is

$$
Z(t) \mid \mathcal{F}_{s} \sim \sum_{i=1}^{2} \mathbb{P}_{(i)}(s, t) N\left(g^{\mathrm{T}} \mu_{(i)}^{Q}(s, t)-H, g^{\mathrm{T}} \Sigma_{(i)}(s, t) g\right)
$$


Hence, by defining $a_{i}(s, t)$ and $c_{i}(s, t)$ as in Eqs. (29)-(30) and $U \sim \mathrm{N}(0,1)$, we obtain

$$
\begin{aligned}
\mathbb{E}^{Q}\left[Z(t)^{+} \mid \mathcal{F}_{s}\right] & =\sum_{i=1}^{2} \mathbb{P}_{(i)}(s, t) \mathbb{E}^{Q}\left[\left(a_{i}(s, t)+c_{i}(s, t) U\right)^{+} \mid \mathcal{F}_{s}\right] \\
& =\sum_{i=1}^{2} \mathbb{P}_{(i)}(s, t) \int_{-a_{i}(s, t) / c_{i}(s, t)}^{\infty} \frac{a_{i}(s, t)+c_{i}(s, t) u}{\sqrt{2 \pi}} e^{-u^{2} / 2} d u \\
& =\sum_{i=1}^{2} \mathbb{P}_{(i)}(s, t)\left(a_{i}(s, t) \Phi\left(\frac{a_{i}(s, t)}{c_{i}(s, t)}\right)+c_{i}(s, t) \phi\left(\frac{a_{i}(s, t)}{c_{i}(s, t)}\right)\right),
\end{aligned}
$$

where $\mathbb{P}_{(i)}(s, t)$ is defined in Eq. (16).

To further comment on Prop. 5, we set $g=\left[\begin{array}{ll}1 & -1\end{array}\right]$, and $H=0$, which is also the most relevant case having the transmission rights in Europe in mind. Consequently, we get that $g^{\mathrm{T}} S(t)-H=0$ in the EPC case, and Eq. (28) thus reduces to

$$
\mathbb{E}^{Q}\left[\left(S_{1}(t)-S_{2}(t)\right)^{+} \mid \mathcal{F}_{s}\right]=\mathbb{P}_{(2)}(s, t)\left(a_{(2)}(s, t) \Phi\left(\frac{a_{(2)}(s, t)}{c_{(2)}(s, t)}\right)+c_{(2)}(s, t) \phi\left(\frac{a_{(2)}(s, t)}{c_{(2)}(s, t)}\right)\right) .
$$

Intuitively, the transmission right value should be increasing when the probability of no convergence, $\mathbb{P}_{(2)}(s, t)$, increases; this is confirmed by Eq. (31). As a result, by assuming no price convergence i.e. $\mathbb{P}_{(2)}(s, t)=1$ - one would simply overestimate the value of the transmission right. Also, having Fig. 7(a) in mind and the empirical setup in Sec. 5.1, there is a significant difference between the reduction of transmission right prices throughout the year, simply due to the time-varying probability of EPC.

\section{Conclusion}

In this paper, we propose a model for the joint behaviour of day-ahead electricity prices in a two-market setup where the market coupling mechanism is taken into account in a continuous time manner. The model is dividable in two parts inspired by the way the market coupling mechanism is effectuated in Europe: The first part captures the exact price convergence, modeled by a latent univariate Gaussian Ornstein-Uhlenbeck process. The second part can be interpreted as capturing the domestic prices set in each individual market area, which we model by a partly latent multivariate Gaussian Ornstein-Uhlenbeck process.

We derive theoretical results of the model, and we discuss the estimation of the model in detail. With regard to the latter, our estimation proposal consists of two assumed independent parts: 1) standard Kalman filtering to estimate the process capturing the domestic prices, and 2) particle filtering to estimate the process capturing the exact price convergence. Both approaches are used in combination with maximum likelihood.

The model is exemplified through a case study where we jointly model German and French hour 21 day-ahead prices. We find a strong seasonal behaviour in the estimated probability of observing exact price convergence. Lastly, we discuss derivative prices implied by the model and show that the implied forward and transmission right prices can be obtained by closedform formulas. The impact of the exact price convergence on forward and transmission right prices can be directly read off from the closed-form formulas. In the example with German and French hour 21 day-ahead prices, the strong seasonality in the probability of observing exact price convergence suggests that 1 ) the spread between forward prices throughout a year varies 
greatly, and 2) with everything else being equal the transmission right prices throughout a year vary greatly.

Future research includes the case where the number of market areas exceeds two. Although we have argued that such case is not relevant given the presently traded instruments, advanced scenario analysis could be derived from such model. Furthermore, it would be interesting to investigate how to incorporate, and the implication of including, spikes. As an example, large movements in opposite directions of two prices would significantly impact the value of a transmission right. However, as a consequence of the market coupling mechanism, such scenarios are very rare, and spikes of the same sign are often observed in adjacent market areas. The real impact of such spikes on e.g. derivatives could therefore be of minor importance. This is also supported by the fact that fewer and fewer spikes are observed, which in turn is possible due to the increased market interconnectedness that potentially eliminates spikes.

\section{Acknowledgements}

The authors are grateful for the valuable comments and discussions provided by the participants attending the Quantitative Methods in Finance 2018 Conference in Sydney. The authors would also like to thank one anonymous reviewer for helpful comments that improved the presentation of this paper.

\section{Disclosure statement}

No conflict of interest to declare.

\section{Funding}

Troels Sønderby Christensen is supported by the Innovation Fund Denmark under Grant 518900117B. 


\section{References}

Abate, G. D., Haldrup, N. (2017). Space-time modeling of electricity spot prices. The Energy Journal, 38, pp. 175-196.

Bachelier, L. (1900). Thorie de la spculation. Ann. Sci. col. Normale Supr., 17, pp. 21-86.

Benth, F. E., Šaltytė Benth, J. (2004). The normal inverse Gaussian distribution and spot price modelling in energy markets. International Journal of Theoretical and Applied Finance, 07, pp. 177-192.

Benth, F. E., Kallsen, J., Meyer-Brandis, T. (2007). A non-Gaussian Ornstein-Uhlenbeck process for electricity spot price modeling and derivatives pricing. Applied Mathematical Finance, 14, pp. $153-169$.

Benth, F. E., Meyer-Brandis, T. (2009). The information premium for non-storable commodities. Journal of Energy Markets, 2, 111-140.

Benth, F. E., Piccirilli, M., Vargiolu, T. (2019). Mean-reverting additive energy forward curves in a Heath-Jarrow-Morton framework. Mathematics and Financial Economics, 13, pp. 543-577.

Borovkova, S., Schmeck, M. D. (2017). Electricity price modeling with stochastic time change. Energy Economics, 63, pp. 51-65.

Douglas, S. M., Popova, J. N. (2011). Econometric Estimation of Spatial Patterns in Electricity Prices. The Energy Journal, 32, pp. 81-105.

Epex Spot, A major step towards market integration, 2019. http://www.epexspot.com/en/ market-coupling (accessed 11 July 2019).

Elia, CWE flow-based, 2019. http://www.elia.be/en/products-and-services/ cross-border-mechanisms/transmission-capacity-at-borders/ flow-based-marktkoppeling-centr-w-europa (accessed 11 July 2019).

Geman, H., Roncoroni, A. (2006). Understanding the fine structure of electricity prices. Journal of Business, 79, pp. 1225-1261.

Girsanov, I. (1960). On transforming a certain class of stochastic processes by absolutely continuous substitution of measures. Theory of Probability 6 Its Applications, 5, pp. 285-301.

Haldrup, N., Nielsen, F. S., Nielsen, M. . (2010). A vector autoregressive model for electricity prices subject to long memory and regime switching. Energy Economics, 32, pp. 1044-1058.

Hinderks, W., Wagner, A., Korn, R. (2018). A structural Heath-Jarrow-Morton framework for consistent intraday, spot, and futures electricity prices. arXiv.

Hubbard., R. A., Inoue, L. Y. T., Fann, J. R. (2014). Modeling nonhomogeneous Markov processes via time transformation. Biometrics, 64, pp. 843-850.

Ichihara, K., Kunita, H. (1974). A classification of the second order elliptic operator and its probabilistic characterization. Z. Wahrsch. Verv. Geb., 30, pp. 235-254.

Ignatieva, K., Trck, S. (2016). Modeling spot price dependence in Australian electricity markets with applications to risk management. Computers $\&$ Operations Research, 66, pp. 415-433. 
International Energy Agency (2017). Monthly electricity statistics. December 2017 report for electricity production and trade data for all OECD member countries.

Inamura, Y. (2006). Estimating continuous time transition matrices from discretely observed data. Bank of Japan Working Paper Series..

Kantas, N., Doucet, A., Singh, S. S., Maciejowski, J., Chopin, N. (2015). On particle methods for parameter estimation in state-space models. Statistical Science, 30, pp. 328-351.

Latini, L., Piccirilli, M., Vargiolu, T.. (2019). Mean-reverting no-arbitrage additive models for forward curves in energy markets. Energy Economics, 79, pp. 157-170.

Laub., A. J. (2005). Matrix Analysis for Scientists and Engineers. Society for Industrial and Applied Mathematics, ISBN: 978-0-898715-76-7.

Lucia, J. J., Schwartz, E. (2002). Electricity prices and power derivatives: evidence from the Nordic power exchange. Review of Derivatives Research, 5, pp. 5-50.

Macedo, H. D., Oliveira, N. O. (2013). Typing linear algebra: A biproduct-oriented approach. Science of Computer Programming, 78, pp. 2160-2191.

Mahringer, S., Füss, R., Prokopczuk, M. (2015). Electricity market coupling and the pricing of transmission rights: an option-based approach. Working papers on Finance 1512. University of St. Gallen, School of Finance.

Meyer-Brandis, T., Tankov, P. (2008). Multi-factor jump-diffusion models of electricity prices. International Journal of Theoretical and Applied Finance, 11, pp. 503-528.

Park, H., Mjelde, J. W., Bessler, D. A. (2006). Price dynamics among U.S. electricity spot markets. Energy Economics, 28, pp. 81-101.

Pircalabu, A., Benth, F. E. (2017). A regime-switching copula approach to modeling day-ahead prices in coupled electricity markets. Energy Economics, 68, pp. 283-302.

Pitt, M. K., Malik, S., Doucet, A. (2014). Simulated likelihood inference for stochastic volatility models using continuous particle filtering. Annals of the Institute of Statistical Mathematics, 66, pp. 527-552.

Schwartz, E., Smith, J. E. (2000). Short-term variations and long-term dynamics in commodity prices. Management Science, 46, pp. 893-911. 